\title{
Fatigue-Sensitive Afferents Inhibit Extensor but Not Flexor Motoneurons in Humans
}

\author{
Peter G. Martin, Janette L. Smith, Jane E. Butler, Simon C. Gandevia, and Janet L. Taylor \\ Prince of Wales Medical Research Institute and University of New South Wales, Randwick, New South Wales 2031, Australia
}

\begin{abstract}
The role of group III and IV muscle afferents in controlling the output from human muscles is poorly understood. We investigated the effects of these afferents from homonymous or antagonist muscles on motoneuron pools innervating extensor and flexor muscles of the elbow. In study 1 , subjects $(n=8)$ performed brief maximal voluntary contractions (MVCs) of elbow extensors before and after a $2 \mathrm{~min}$ MVC of the extensors. During MVCs, electromyographic responses from triceps were evoked by stimulation of the corticospinal tracts [cervicomedullary motor evoked potentials (CMEPs)]. The same subjects repeated the protocol, but input from fatigue-sensitive afferents was prolonged after the fatiguing contraction by maintained muscle ischemia. In study 2, CMEPs were evoked in triceps during brief extensor MVCs before and after a 2 min sustained flexor MVC $(n=7)$ or in biceps during brief flexor MVCs before and after a sustained extensor MVC $(n=7)$. Again, ischemia was maintained after the sustained contractions. During sustained MVCs of the extensors, CMEPs in triceps decreased by $\sim 35 \%$. Without muscle ischemia, CMEPs recovered within $15 \mathrm{~s}$, but with maintained ischemia, they remained depressed (by $\sim 28 \% ; p<0.001$ ). CMEPs in triceps were also depressed (by $\sim 20 \% ; p<0.001$ ) after fatiguing flexor contractions, whereas CMEPs in biceps were facilitated (by $\sim 25 \% ; p<0.001$ ) after fatiguing extensor contractions. During fatigue, inputs from group III and IV muscle afferents from homonymous or antagonist muscles depress extensor motoneurons but facilitate flexor motoneurons. The more pronounced inhibitory influence of these afferents on extensors suggests that these muscles may require greater cortical drive to generate force during fatigue.
\end{abstract}

Key words: fatigue; motoneuron; group III and IV muscle afferents; human; corticospinal; ischemia

\section{Introduction}

During a sustained isometric maximal voluntary contraction (MVC), the discharge rate of motoneurons declines. Although observed in several human muscles (Grimby et al., 1981; BiglandRitchie et al., 1983; Gandevia et al., 1990), the cause of the decline is controversial. It is widely believed that activity in group III and IV muscle afferents, which are sensitive to ischemia and metabolites, inhibits spinal motoneurons (for review, see Garland and Kaufman, 1995). When firing of group III and IV muscle afferents is maintained after fatiguing exercise by muscle ischemia, the recovery of the discharge rates of motoneurons is prevented (Bigland-Ritchie et al., 1986; Woods et al., 1987). The tendon jerk and H-reflex are also reduced by fatigue (Garland and McComas, 1990; Garland, 1991; Duchateau and Hainaut, 1993; Walton et al., 2002; Kuchinad et al., 2004), and a contraction-induced spinal inhibition may operate between synergists (Sacco et al., 1997). These studies suggest that group III and IV muscle afferent feedback from the fatiguing muscle can reflexly depress or inhibit motoneuronal firing rates. However, maintained muscle ischemia does not affect the recovery of responses produced by stimulation of the corticospinal tract after a sustained maximal con-

Received Dec. 22, 2005; revised Feb. 27, 2006; accepted March 18, 2006.

This work was supported by the National Health and Medical Research Council of Australia.

Correspondence should be addressed to Dr. Janet Taylor, Prince of Wales Medical Research Institute, University of New South Wales, Barker Street, Randwick, Sydney, New South Wales 2031, Australia. E-mail: jl.taylor@unsw.edu.au.

DOI:10.1523/JNEUROSCI.5487-05.2006

Copyright $\odot 2006$ Society for Neuroscience $\quad$ 0270-6474/06/264796-08\$15.00/0 traction in elbow flexor muscles (Taylor et al., 2000; Butler et al., 2003). This suggests paradoxically, that during fatigue, group III and IV muscle afferents do not directly inhibit elbow flexor motoneurons.

In the cat, group III and IV muscle afferents have different actions on flexor and extensor motoneuron pools (Kniffki et al., 1979, 1981a,b; Schomburg et al., 1999). Direct measurements from $\alpha$ motoneurons during chemical activation of afferents of an extensor muscle showed that many motoneurons innervating extensor muscles were hyperpolarized, whereas those innervating flexors were depolarized, although these effects were not uniform. The effect of afferents from a flexor muscle on motoneurons was not investigated. Excitation of flexor muscles with inhibition of extensors is consistent with the predominant actions evoked by several classes of afferents collectively referred to as flexor reflex afferents (FRAs) (Eccles and Lundberg, 1959). However, FRAs also have alternate excitatory and inhibitory reflex pathways under different conditions (Holmqvist and Lundberg, 1961). In humans, it is not known whether the actions of group III and IV afferents differ between motoneuron pools. Furthermore, it is not known whether the same reflex effect is exerted by fatigue-sensitive afferents from flexor and extensor muscles.

In the present study, motoneuronal excitability was assessed by stimulation of the descending corticospinal pathways at the cervicomedullary level during maintained muscle ischemia after fatiguing exercise. Given that the motoneuron pool of the elbow flexor muscles is not inhibited by the firing of homonymous fatigue-sensitive afferents, the current study aimed to define the 
reflex effects of group III and IV muscle afferents on the motoneurons of the elbow extensor muscles and to determine whether afferents activated by fatigue of the flexor and extensor muscles had similar actions.

\section{Materials and Methods}

In a series of experiments, corticospinal tract stimulation was used to assess the influence of group III and IV muscle afferent inputs from homonymous or antagonist muscles on the behavior of the motoneuron pools innervating the extensor and flexor muscles of the elbow. A total of 10 healthy adult volunteers (three females) participated. The first set of studies was performed on eight subjects who were each studied on two occasions. Seven subjects completed the second set of studies, which also involved two sets of experiments. Five of the subjects performed both of these experiments. A third study, involving four subjects who had participated in at least one of the experiments from studies 1 and 2, was performed to assess changes in blood pressure. The procedures were approved by the local ethics committee, and the study was conducted according to the Declaration of Helsinki. All subjects gave their informed consent to participate.

Many of the experimental procedures and recording techniques were similar to those described previously (Butler et al., 2003). The subject was seated with the right shoulder flexed to $90^{\circ}$, the elbow flexed to 75 or $80^{\circ}$, and the forearm supinated. The arm was held in an isometric myograph with a strap at the wrist. Fatigue was induced in either the elbow extensor or flexor muscles by a sustained 2 min MVC. Subjects received visual feedback of torque and were encouraged to perform maximally throughout all contractions. EMG was recorded from either triceps brachii or from biceps brachii and brachioradialis via self-adhesive electrodes (Ag$\mathrm{AgCl} ; 10 \mathrm{~mm}$ diameter) placed $\sim 5 \mathrm{~cm}$ apart over the midbelly of each muscle with the distal electrode close to the tendon. The signals were amplified, filtered $(16-1000 \mathrm{~Hz})$, and collected at $2 \mathrm{kHz}$ for off-line analysis using customized software (CED 1902 amplifiers; CED 1401 with Signal and Spike2 software; Cambridge Electronic Design, Cambridge, UK). During MVCs, stimuli were delivered to the brachial plexus or to the corticospinal tract, and EMG responses were recorded.

\section{Stimulation}

Brachial plexus stimulation. Stimuli were delivered to the brachial plexus at Erb's point so that the maximal $M$ wave could be monitored $\left(M_{\max }\right)$. The stimulus intensity $(68-270 \mathrm{~mA})$ was at least $50 \%$ above that required to produce a maximal response in triceps or biceps. Stimuli were rectangular pulses (100 $\mu$ s duration) delivered with the cathode in the supraclavicular fossa and the anode over the acromion (Digitimer DS7 constant-current stimulator; Digitimer, Welwyn Garden City, UK).

Corticospinal tract stimulation. The corticospinal tract was stimulated by passing a high-voltage electrical current (100 $\mu$ s duration; Digitimer D180) between cup electrodes filled with conductive gel and glued to the skin (1-2 cm posterior and superior to the tip of the mastoid processes with the cathode on the left side) (Ugawa et al., 1991; Gandevia et al., 1999). Activation occurs at the cervicomedullary junction and evokes large, short-latency responses [cervicomedullary motor evoked potentials (CMEPs)] in arm muscles. The stimulus intensity ranged between 413 and $694 \mathrm{~V}$ to evoke responses with an area of $\sim 50 \% M_{\max }$ during brief control MVCs. At this intensity, the latency of responses during MVCs was $7.9 \pm 0.7 \mathrm{~ms}$ (mean $\pm \mathrm{SD}$ ), $8.0 \pm 0.5 \mathrm{~ms}$, and $10.4 \pm 0.4 \mathrm{~ms}$ for triceps, biceps, and brachioradialis, respectively. The latency of responses was monitored carefully to ensure that high stimulation intensities did not activate the motor axons at or near the ventral roots. A jump in latency of $\sim 2$ ms occurs when the site of stimulation spreads from descending tracts to the ventral roots (Taylor and Gandevia, 2004).

\section{Experimental tasks and recordings}

Study 1: experiments to investigate effects of fatigue-sensitive afferents from homonymous muscles on elbow extensor motoneurons. In these experiments, the elbow was fixed at $75^{\circ}$ flexion. The test fatiguing contraction was a sustained MVC of the right elbow extensors lasting $120 \mathrm{~s}$. Before the sustained contraction, six brief MVCs of the extensors (each lasting 1-2 s) were performed at $\sim 1 \mathrm{~min}$ intervals. After the sustained contraction, brief MVCs of the extensors were performed (at 15, 30, 45, 60, 75, 90, 120, $135,180,195,240,255,300$, and $315 \mathrm{~s}$ after the sustained test contraction) to assess the recovery of the EMG responses to stimulation. This protocol was repeated on another day with one change: a sphygmomanometer cuff around the upper arm was inflated to $300 \mathrm{mmHg}$ from a source of compressed air $10 \mathrm{~s}$ before the end of the sustained contraction. The cuff had a proximal and distal compartment, of which only the proximal compartment was inflated. This meant that the inflated region was proximal to the EMG electrodes. Inflation of the cuff was maintained for $120 \mathrm{~s}$ into the recovery period, during which eight brief MVCs were performed at $15 \mathrm{~s}$ intervals. After the cuff was deflated, the subjects performed brief recovery MVCs (at 15, 30, 45, 60, 75, 90, 120, 135, 180, 195, $240,255,300,315,360$, and $375 \mathrm{~s}$ after cuff deflation). The two sets of experiments were performed several days apart, and their order was randomized between subjects.

Stimuli to either the brachial plexus or the corticospinal tracts were delivered alternately at the peak force of each brief MVC to evoke responses in triceps. During the sustained maximal extensions, corticospinal tract and brachial plexus stimuli were delivered in pairs ( $5 \mathrm{~s}$ apart) every $15 \mathrm{~s}$ throughout the 2 min contraction with the first stimulus occurring $2 \mathrm{~s}$ after the start of the contraction.

Study 2: experiments to investigate effects of fatigue-sensitive afferents from the antagonist muscles on elbow flexor and extensor motoneurons. In one experiment, subjects performed brief MVCs of the elbow flexors before and after a sustained MVC of the elbow extensors lasting $120 \mathrm{~s}$. EMG responses to stimulation were recorded from biceps and brachioradialis. In another experiment, brief MVCs of the elbow extensors were performed before and after a sustained MVC of the elbow flexors. Responses were recorded from triceps. Sets performed before and after the sustained MVC were identical and involved eight brief MVCs separated by $15 \mathrm{~s}$ intervals. The sphygmomanometer cuff was inflated with $10 \mathrm{~s}$ remaining in the sustained contraction and remained inflated during the eight brief contractions. After deflation of the cuff, an additional eight brief MVCs were performed at $15 \mathrm{~s}$ intervals. All contractions were performed with the elbow flexed at $80^{\circ}$.

Stimuli to either the brachial plexus or the corticospinal tracts were delivered alternately at the peak force of each brief MVC. Stimuli were not delivered during the sustained MVC.

Study 3: additional control experiments. Fatiguing voluntary contractions are well known to produce reflex increases in blood pressure through the firing of group III and IV afferents (for review, see Kaufman and Forster, 1996). Maintenance of muscle ischemia by inflating a sphygmomanometer cuff around the upper arm after a 2 min maximal elbow flexion produces a reflex increase in blood pressure (Taylor et al., 2000). However, to ensure that a 2 min sustained maximal elbow extension with maintained muscle ischemia induced similar changes, additional experiments were performed. Mean arterial blood pressure was measured noninvasively at the digital arteries of the middle finger on the left (noncontracting) side (Finapres Medical Systems, Amsterdam, The Netherlands) before, during, and after 2 min sustained maximal contractions of the elbow extensors.

\section{Analysis}

Throughout this study, the area of the CMEPs is reported, although similar results occurred if the peak-to-peak amplitude was used. The areas of the CMEPs and $M_{\max }$ were measured between cursors appropriately positioned for each potential. The cursors encompassed a region from the initial deflection from baseline to the second crossing of the horizontal axis (see Fig. 1, top left $M_{\max }$ trace, shaded region). Where possible, each CMEP was normalized to the mean of $M_{\max }$ elicited before and after it (Butler et al., 2003). However, the first CMEP during the sustained MVC and the first CMEPs during and after the period of cuff inflation (and the corresponding CMEPs during the experiment without the cuff) were normalized to the immediately following $M_{\max }$. Thus, CMEPs were normalized to $M_{\max }$ elicited under similar conditions (i.e., with or without the cuff inflated).

Group data are presented as means \pm SD in the text, and the means \pm SEM are shown in the figures (with $n$ in the legends). One-way repeated measures ANOVAs were performed (with Student-Newman-Keuls post 
hoc tests) to assess the differences in responses between different time periods. In the experiments investigating extensor motoneurons following a sustained MVC of the extensors, four time periods (the three control responses, the three responses in the first $40 \mathrm{~s}$ of the sustained MVC, the three responses in the last $40 \mathrm{~s}$ of the sustained MVC, and the four responses in the first $120 \mathrm{~s}$ of recovery) were used in analysis of the data from the experiment without maintained muscle ischemia. An additional time period (the first $90 \mathrm{~s}$ after cuff deflation) was added for analysis of experiments with maintained muscle ischemia. For the subsequent experiments investigating extensor and flexor motoneurons, three time periods were used (the four control responses, the four responses during cuff inflation, and the four responses after cuff deflation). Mean arterial pressure was averaged over $15 \mathrm{~s}$ intervals for each subject, and differences between six time periods (before MVC, first $15 \mathrm{~s}$ during MVC, last $15 \mathrm{~s}$ during MVC, first $15 \mathrm{~s}$ after MVC with the cuff inflated, last $15 \mathrm{~s}$ after MVC with the cuff inflated, and first $15 \mathrm{~s}$ after cuff deflation) were assessed using a one-way repeated-measures ANOVA (with Student-NewmanKeuls post hoc tests). Statistical significance was set at $p<0.05$.

\section{Results}

\section{Study 1}

During the 2 min sustained MVC of the elbow extensors, there were changes in the size of the responses in triceps brachii produced both by corticospinal stimulation (CMEPs) and brachial plexus stimulation $\left(M_{\max }\right)$ (Fig. 1). $M_{\max }$ increased in area compared with responses evoked during the brief control MVCs. It eventually declined late in the contraction but remained elevated compared with the initial control values $(p<0.001)$. The CMEP also increased slightly during the first part of the contraction and declined to be slightly smaller by the completion of the sustained MVC, but these changes were not significant (Fig. 2A). Thus, relative to $M_{\max }$, the CMEP decreased during the fatiguing contraction.

Because changes in $M_{\max }$ must also influence the CMEP (Taylor et al., 1999), all responses to cervicomedullary stimulation were normalized to $M_{\max }$ elicited under similar conditions (see Materials and Methods). The normalized CMEP decreased in area during the 2 min MVC and in the first set of experiments, without maintained muscle ischemia, recovered quickly to control levels. The area of the CMEP was initially $49 \pm 10 \% M_{\max }$ during the brief MVCs and decreased to $33 \pm 14 \% M_{\max }$ late in the sustained contraction $(p<0.005)$ (Fig. $2 B)$. Responses during the brief recovery contractions $\left(45 \pm 12 \% M_{\max }\right)$ were not significantly different from control values $(p=0.329)$.

To test whether group III and IV muscle afferents contributed to the decrease in size of the normalized CMEP during the $2 \mathrm{~min}$ maximal contraction of the elbow extensors, the experiment was repeated in the same subjects but with the muscles held ischemic for an additional $2 \mathrm{~min}$ after the end of the sustained contraction (Fig. $2 C, D$ ). If group III and IV muscle afferents contributed to the decrease in the CMEP during extension, this decrease should be maintained throughout ischemia. When the muscles were not held ischemic, the CMEP decreased relative to $M_{\max }$ during the 2 $\min$ MVC from $53 \pm 9 \% M_{\max }$ to $31 \pm 13 \% M_{\max }(p<0.001)$. However, unlike the first experiment, when the muscle was held ischemic, the CMEP remained depressed during the first 2 min of recovery. During this period, the CMEP recovered slightly to $38 \pm$ $15 \% M_{\max }(p=0.067)$ but was still significantly reduced compared with control values ( $p=0.001$ ) (Fig. $2 D)$. Once the cuff was deflated, responses returned to near control levels within $15 \mathrm{~s}$. This suggests that the maintained muscle ischemia affected the recovery of the CMEP elicited during brief maximal contractions of elbow extensors after the sustained contraction. In a previous study, maintained muscle ischemia did not affect the recovery of the CMEP in the elbow flexors after fatigue (Butler et al., 2003).

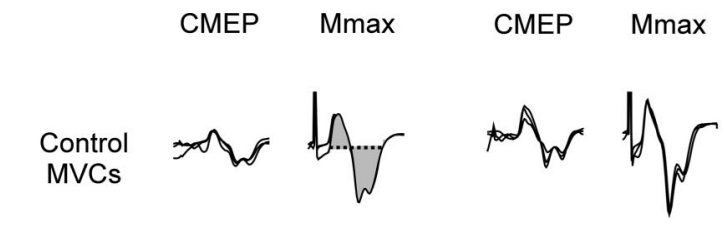

$2 \min$ MVC

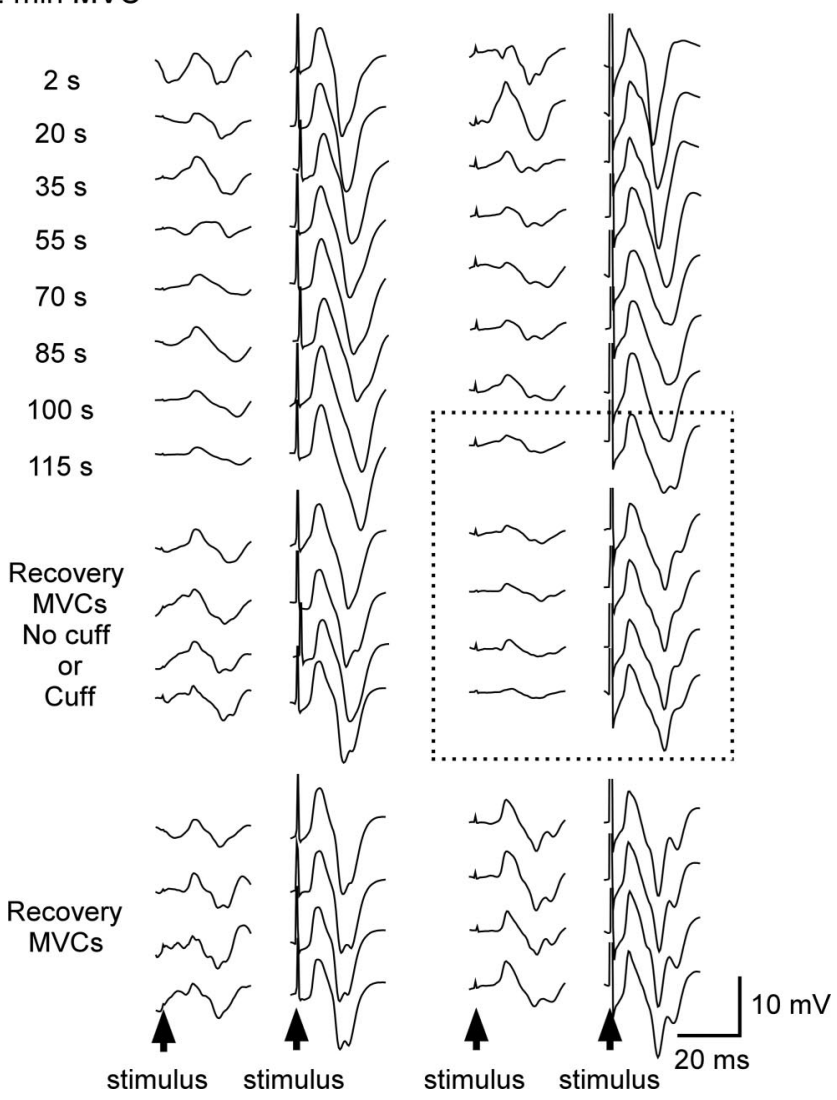

Figure 1. EMG responses in triceps brachii of a typical subject before, during, and after a sustained MVC of the elbow extensors without and with subsequent maintained muscle ischemia. Single responses to stimulation of the corticospinal tract (CMEP) and the brachial plexus $\left(M_{\max }\right)$ are shown. All responses were obtained during MVCs of the elbow extensors. The two left columns of traces were recorded in one experiment. At the top of each column, control responses evoked during brief MVCs are overlaid. The next eight traces were recorded during a 2 min extensor MVC. The time of corticospinal tract stimulation is indicated on the left, with brachial plexus stimulation $5 \mathrm{~s}$ later. The next four traces were recorded during brief MVCs in the first $2 \mathrm{~min}$ after the end of the sustained MVC and the final four traces in an additional $3 \mathrm{~min}$. The two right columns of traces were recorded from the same subject on a different day. Here, a cuff inflated around the upper arm held the muscle ischemic after the sustained contraction. The period of ischemia is indicated by a dotted box. For each column of traces, timing of the stimulus is shown by an arrow. The shaded region shown for the top $M_{\max }$ traces indicates the area used for analysis. The area of the CMEP was measured similarly.

\section{Study 2}

The differences in the behavior of the CMEP recorded from the extensor and flexor muscles during maintained muscle ischemia after fatigue could be a result of either motoneuron type (flexor vs extensor motoneurons) or the source of the afferent input (flexor vs extensor muscles). Hence, in separate experiments, we selectively fatigued the elbow extensors or flexors and evoked CMEPs in the antagonist muscles. The firing of group III and IV muscle afferents from the fatigued muscle was maintained by muscle ischemia. $M_{\max }$ in the nonfatigued muscle was not significantly affected in either experiment $(p>0.05)$. After a sustained MVC of the elbow flexors, the normalized CMEP evoked in triceps 
A
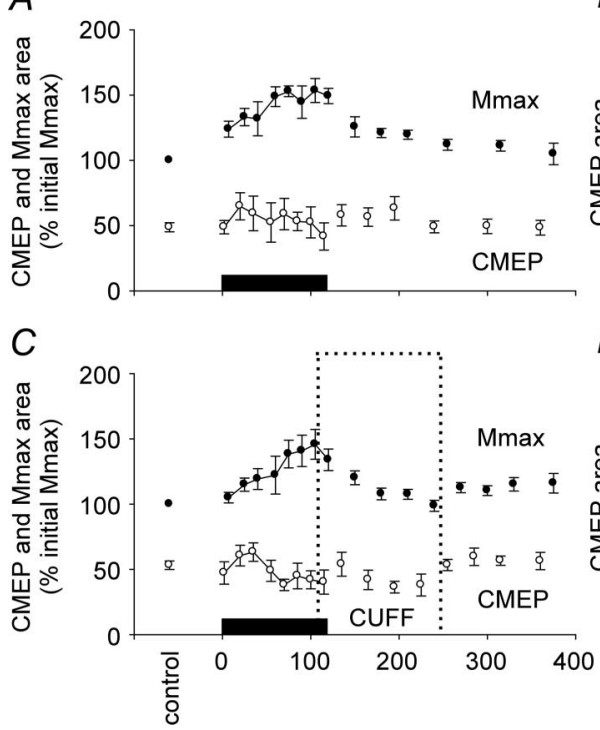

B

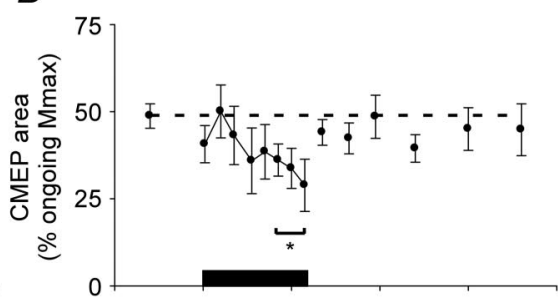

$D$

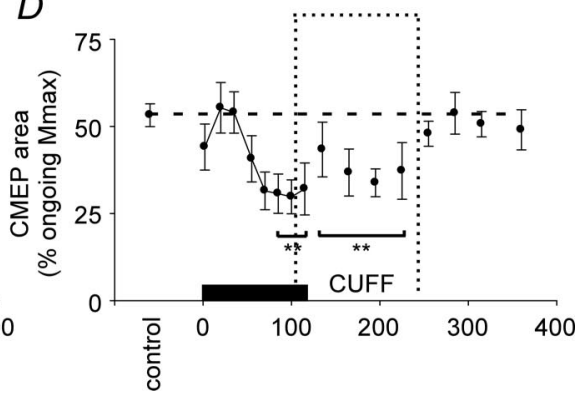

Figure 2. Group data showing the area of EMG responses in triceps brachii before, during, and after a sustained MVC of the elbow extensors without and with subsequent maintained muscle ischemia ( $n=8$ subjects). All responses were evoked during brief or sustained extensor MVCs. In each panel, the solid bar indicates the timing of the sustained MVC. Top panels show data from experiments in which muscle ischemia was not maintained. Bottom panels show data recorded when muscle ischemia was maintained over the period indicated by the dotted box (CUFF). $\boldsymbol{A}$ and $\boldsymbol{C}$ show the mean area ( $\pm \mathrm{SEM}$ ) of the responses to stimulation of the corticospinal tract (CMEP; open circles) and the brachial plexus ( $M_{\text {max }}$; filled circles) expressed as a proportion of the mean initial maximal $M$-wave (\% initial $M_{\text {max }}$ ). $\boldsymbol{B}$ and $\boldsymbol{D}$ show the same data as in $\boldsymbol{A}$ and $\boldsymbol{C}$ but with the area of the CMEP normalized to $M_{\max }$ recorded at close to the same time (\% ongoing $M_{\max }$ ) to account for any change in the muscle fiber action potential. The dashed horizontal lines indicate the mean area of CMEPs during brief control MVCs. During the 2 min MVC, the CMEP decreases. In the absence of ischemia, it recovers quickly to control size, whereas during maintained ischemia, it remains depressed. * ${ }^{*}$ CMEPs in the last 40 s of sustained MVC were reduced compared with those in the control period, first 40 s of sustained MVC, and first 120 s of recovery $(p<0.05) .{ }^{* *}$, CMEPs in the last 40 s of sustained MVC and during muscle ischemia were reduced compared with those in the first $40 \mathrm{~s}$ of sustained MVC and first $90 \mathrm{~s}$ after cuff deflation $(p<0.005)$.

during brief MVCs of the elbow extensors was significantly reduced compared with control values. Responses were initially $47 \pm 8 \% M_{\max }$ and were reduced to $38 \pm 8 \% M_{\max }$ during the period of maintained muscle ischemia $(p<0.001)$ (Figs. 3, left traces, 4 , closed symbols). Responses recovered quickly to control values on cuff deflation. Conversely, when the elbow extensors were fatigued and the CMEP evoked during brief MVCs of the elbow flexors, responses were facilitated during maintained ischemia. The CMEP in biceps brachii was initially $52 \pm 8 \% M_{\max }$ and increased in area to $65 \pm 9 \% M_{\max }$ during maintained ischemia $(p<0.01)$ (Figs. 3, right traces, 4 , open symbols). The CMEP in brachioradialis also increased from $42 \pm 13 \% M_{\max }$ to $51 \pm 9 \% M_{\max }(p<0.05)$. The CMEP in both biceps and brachioradialis returned to control values within $15 \mathrm{~s}$ of cuff deflation. These results suggest that group III and IV muscle afferents from the fatigued muscles project onto their antagonist muscles and that the motoneurons of extensor and flexor muscles are differentially affected by these inputs. Figure $5 A$ summarizes the effect of a period of maintained muscle ischemia after fatigue on CMEPs evoked during brief contractions of the extensors or flexors. Relative to its size during brief control MVCs, the CMEP evoked in triceps was reduced after a fatiguing extension (Fig. $5 A$, white column) or after a fatiguing flexion (Fig. $5 A$, gray column), whereas the CMEP evoked in biceps was facilitated after a fatiguing extension (Fig. 5A, black column). In a previous study (Butler et al., 2003), there was a nonsignificant facilitation of the CMEP evoked in biceps after a sustained flexion (Fig. 5A, striped column).
Study 3

Mean arterial pressure increased during the sustained maximal elbow extension by $\sim 50 \mathrm{mmHg}(p<0.001)$ and plateaued after $\sim 60 \mathrm{~s}$. Blood pressure declined immediately as the contraction ceased but remained significantly elevated above control levels (by $\sim 20 \mathrm{mmHg}$ ) as long as the cuff was inflated $(p<0.01)$. It returned to control levels within $15 \mathrm{~s}$ of cuff deflation $(p=0.303)$.

\section{Discussion}

These studies provide new data on the effects of group III and IV muscle afferents from homonymous and antagonist muscles on motoneuron pools innervating extensor and flexor muscles of the human elbow. During a 2 min MVC of the elbow extensors, CMEPs recorded in triceps brachii decreased by $\sim 30-40 \%$ when normalized to $M_{\max }$. This is consistent with the behavior of CMEPs during a fatiguing contraction of the elbow flexors (Taylor et al., 2000; Butler et al., 2003). However, unlike the flexors, CMEPs evoked in extensor muscles do not recover when ischemia is maintained after fatiguing contractions. This suggests that ischemically sensitive small-diameter muscle afferents inhibit the motoneurons of elbow extensors during fatigue.

The reduction in the size of CMEPs with fatigue is consistent with a decrease in the excitability of motoneurons. Because CMEPs returned to control levels in the absence of ischemia, it is unlikely that the depression of CMEPs during the sustained MVC was caused by either a reduced efficacy of the motoneuronal synapse or activity-dependent changes in corticospinal axons. The time course of recovery of both effects means that if either were occurring during the sustained MVC, it should continue during the brief MVCs in the recovery period (Gandevia et al., 1999). The reduced motoneuronal excitability probably contributes to the decline in the discharge rate of single motor units and the development of central fatigue that occurs with sustained MVCs. The reduction in motoneuron firing rates observed in many studies (Grimby et al., 1981; Bigland-Ritchie et al., 1983; Gandevia et al., 1990) has often been attributed to reflex inhibition from group III and IV muscle afferents (Woods et al., 1987; Garland, 1991). In the current study, when ischemia was maintained after fatiguing contractions of elbow extensors, CMEPs in triceps failed to recover. The continued elevation of mean arterial blood pressure during the ischemia indicates that the firing of group III and IV muscle afferents was maintained. When perfusion was restored to the muscle, both arterial pressure and CMEPs recovered to prefatigue levels within $15 \mathrm{~s}$. Therefore, some of the reduction in extensor CMEPs during the MVC probably relates to firing of group III and IV muscle afferents. In contrast, although CMEPs are also depressed during fatiguing MVCs of elbow flexors, this depression recovers despite maintained muscle ischemia suggesting that elbow flexor motoneurons are not directly inhibited by group III and IV muscle afferents (Taylor et al., 2000; Butler et al., 2003). Together, these 


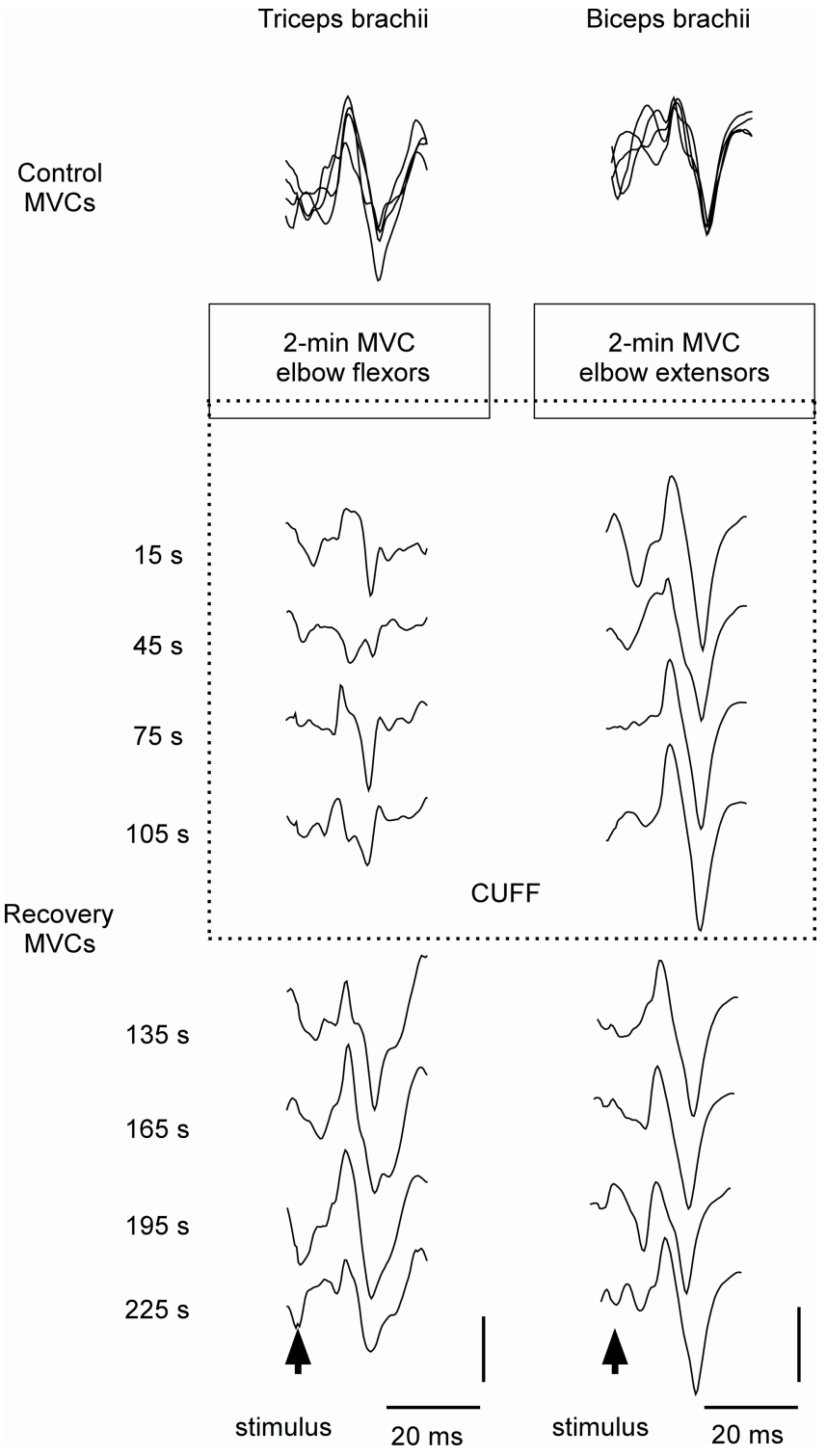

Figure 3. CMEPs recorded from triceps brachii and biceps brachii before and after $2 \mathrm{~min}$ MVCs of their antagonist muscles. The left column of traces shows responses to corticospinal tract stimulation (CMEPs) evoked in triceps during brief MVCs of the elbow extensors before (controls, 4 overlaid traces) and after a 2 min maximal elbow flexion. For 2 min after the sustained MVC, an inflated cuff maintained muscle ischemia (dotted box labeled CUFF; middle 4 traces). The right column of traces shows CMEPs recorded from biceps in the same subject before and after a 2 min elbow extension. The dotted box again indicates a period of maintained muscle ischemia. The arrow indicates the timing of the stimulus for each column of traces. The vertical scale bar for the left column represents $5 \mathrm{mV}$, and in the right column it represents $10 \mathrm{mV}$.

results suggest differential influences of group III and IV muscle afferents on different motoneuron pools.

The different effects of group III and IV muscle afferents on motoneurons of elbow extensors and flexors in humans (Taylor et al., 2000; Butler et al., 2003) is consistent with results in the cat. Direct measurements from motoneurons during chemical stimulation of group III and IV muscle afferents in extensor muscles showed that many extensor motoneurons were hyperpolarized, whereas many motoneurons innervating flexor muscles depolarized (Kniffki et al., 1979, 1980, 1981a,b). The pattern of activation is also consistent with the predominant actions of FRAs (Eccles and Lundberg, 1959). In acute spinal cats, however, a reversed pattern of flexor inhibition or extensor excitation was observed

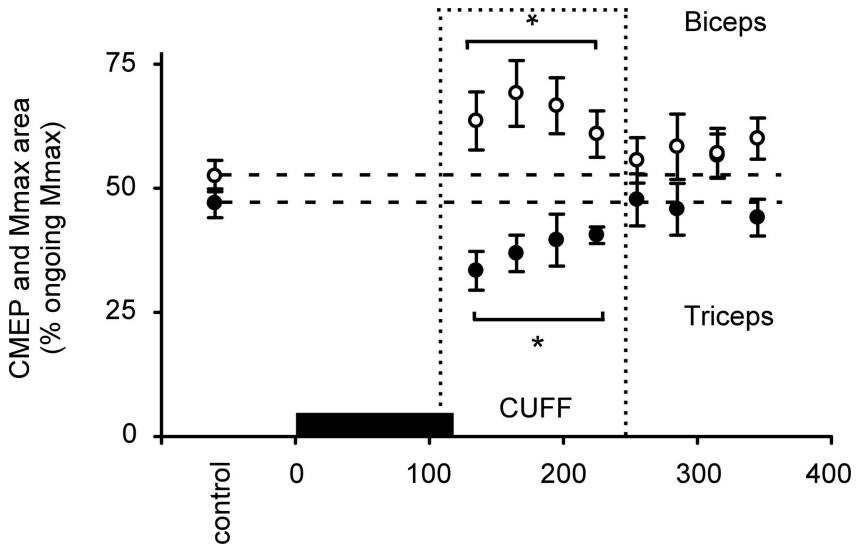

Figure 4. Pooled data for CMEPs evoked during brief MVCs before and after a 2 min MVC of the antagonist muscles. CMEPs were evoked in triceps (filled circles; mean \pm SEM; $n=7$ ) during brief extensor MVCs before and after a 2 min elbow flexion. CMEPs were evoked in biceps (open circles; $n=7$ ) during brief flexor MVCs before and after a 2 min elbow extension. The solid bar represents the timing of the sustained maximal effort. A cuff inflated near the end of the sustained effort maintained muscle ischemia for $2 \mathrm{~min}$ (dotted box labeled (UFF). The area of each CMEP is normalized to $M_{\max }$ recorded at close to the same time. The dashed horizontal lines indicate the mean areas of the triceps and biceps CMEPs during brief control MVCs. * CMEPs during ischemia are significantly different to control and postcuff values $(p<0.05)$.

under some conditions, indicating that there are also "private" paths apart from the flexion reflex (Holmqvist and Lundberg, 1961). Additionally, in animals (Levinsson et al., 1999) as well as the human lower limb (Hagbarth, 1960; Andersen et al., 1999), actions evoked by specific cutaneous afferents depended on the location of stimulation. Our results suggest that such alternative paths do not dominate during fatiguing contractions in humans.

In studies of the cat, afferents from extensor muscles exerted different effects on motoneurons of flexors compared with extensors (Kniffki et al., 1979, 1980, 1981a,b). Although the effects of flexor afferent inputs were not tested, the results suggested that the differing behavior related to the type of motoneuron rather than the source of afferent. To clarify the mechanism of differences between flexor and extensor behavior during fatigue in the human arm, we selectively fatigued the elbow extensors or flexors and evoked CMEPs in antagonist muscles. The CMEPs evoked in extensors were again inhibited during maintained ischemia (by $\sim 20 \%$ ), this time by afferents from the fatigued flexors. Conversely, fatigue-sensitive afferents from extensors facilitated responses in flexors (by $\sim 25 \%$ ). These results indicate that group III and IV muscle afferents project onto antagonist muscles of the elbow, and that the differential behavior of flexor and extensor motoneuron pools does not relate to the source of afferent input (Fig. 5B).

In this study, motoneuronal excitability was tested indirectly by stimulation of the corticospinal path at the cervicomedullary level. In humans, descending pathways are thought to be free from traditional presynaptic control (Nielsen and Petersen, 1994). For biceps, a significant proportion of the motoneuronal response is monosynaptic (Petersen et al., 2002). Although corticospinal connections to triceps are weaker than for biceps (Palmer and Ashby, 1992; de Noordhout et al., 1999), evidence favors a primarily monosynaptic corticomotoneuronal connection even to triceps (de Noordhout et al., 1999). Therefore, changes in responses evoked in flexors and extensors probably reflect effects at a motoneuronal level.

Apart from direct inhibition or facilitation of motoneurons, group III and IV muscle afferents may modulate motoneuron firing indirectly. First, they may inhibit group Ia terminals pre- 
$A$
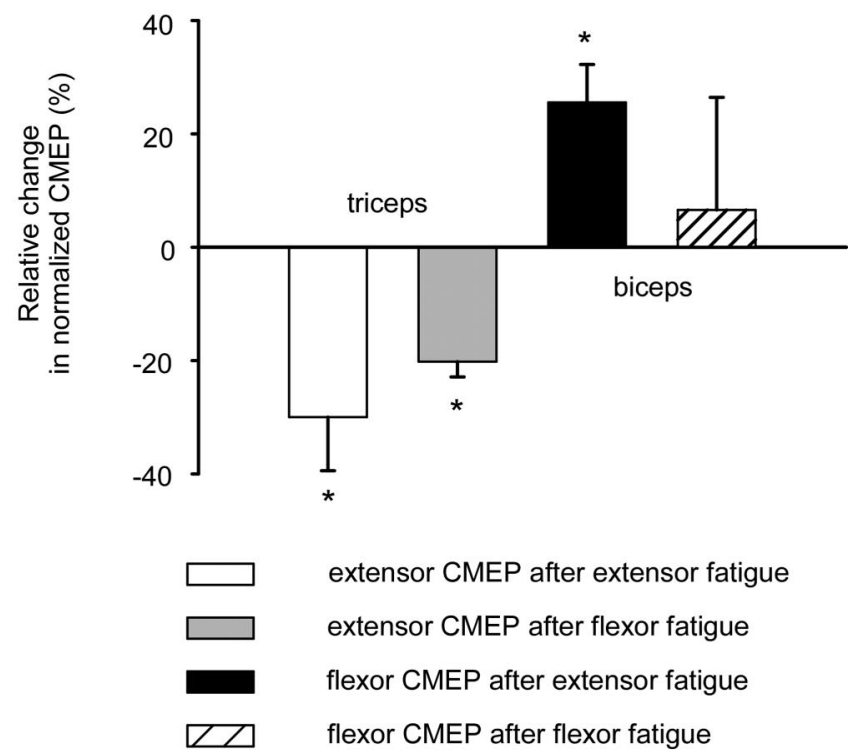

$B$

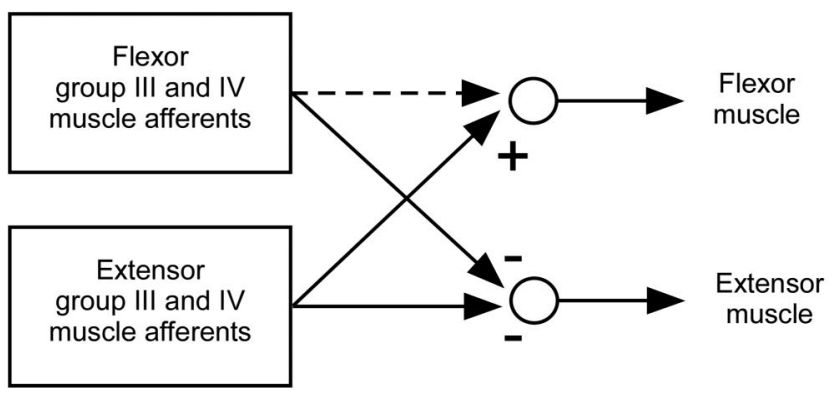

Figure 5. Summary of changes in the CMEP produced by maintained muscle ischemia after a fatiguing contraction. $\boldsymbol{A}$, Change in the CMEP in triceps or biceps during maintained muscle ischemia after a 2 min MVC of the elbow extensors or flexors. The difference between the area of the normalized CMEP during maintained ischemia and in brief control MVCs is expressed as a percentage of its control size. That is, the percentage facilitation or depression of the CMEP is shown. CMEPs in triceps were always evoked during brief extension MVCs and CMEPs in biceps during brief flexion MVCs. The four columns represent changes in different experiments: (1) the CMEP was evoked in triceps before and after a 2 min extensor MVC (white); (2) the CMEP in triceps before and after a 2 min flexor MVC (gray); (3) the CMEP in biceps before and after a 2 min extensor MVC (black); and (4) the CMEP in biceps before and after a 2 min flexor MVC (right to left shading) (Butler et al. 2003). ${ }^{*}$, Significant difference between CMEPs during maintained ischemia and before the sustained MVC. $\boldsymbol{B}$, Schematic diagram showing apparent effects of firing of group III and IV muscle afferents on the flexor and extensor motoneuron pools. Findings in the current study indicate facilitation of flexors by extensor afferents and inhibition of extensors by flexor or extensor afferents. A previous study (Butler et al., 2003) showed nonsignificant facilitation of the flexors by flexor afferents (dashed line).

synaptically and thus disfacilitate motoneurons (Pettorossi et al., 1999; Rossi et al., 1999). However, other factors could counteract such effects. In the cat, group III and IV muscle afferents can increase fusimotor discharge (Jovanovic et al., 1990) and hence spindle discharge during muscle fatigue (Ljubisavljevic et al., 1992). Second, group III and IV afferents may alter recurrent inhibition. In the cat, Renshaw cells tended to become less responsive (Windhorst et al., 1997; Kalezic et al., 2004), whereas in humans, recurrent inhibition was increased (Kukulka et al., 1986; Rossi et al., 2003). Finally, group III and IV muscle afferents may mediate changes in motoneuron afterhyperpolarization during fatigue; however, at least in the cat, this does not slow motoneuron discharge (Windhorst et al., 1997). Therefore, it is likely that small-diameter muscle afferents exert both facilitatory and inhibitory effects on motoneurons during fatigue. Our findings indicate that the balance of these inputs inhibits extensors but facilitates flexor motoneurons in the human arm.

The differential effect of small-diameter afferents activated by fatigue on motoneurons innervating extensors and flexors may not be unique to human elbow muscles. Much of the indirect evidence favoring a reflex inhibitory pathway mediated by group III and IV muscle afferents has been obtained for other physiological extensors. For example H-reflexes evoked in ankle plantarflexors are depressed after fatigue (Garland and McComas, 1990; Garland, 1991; Loscher et al., 1996; Walton et al., 2002; Kuchinad et al., 2004). We suggested previously that the discrepancy between results based on the H-reflex and that for the CMEP in elbow flexors (Butler et al., 2003) could be attributable to presynaptic inhibition of group Ia afferent volleys by group III and IV muscle afferents (Pettorossi et al., 1999; Rossi et al., 1999; Rudomin and Schmidt, 1999). The current results suggest that differences in the behavior of flexors and extensors may also contribute.

During maintained muscle ischemia after fatigue, CMEPs evoked in elbow flexors recover, but there was also a partial recovery of CMEPs evoked in elbow extensors. The CMEP in triceps recovered approximately one-third of its fatigue-related depression during maintained ischemia after sustained extensions. These results suggest that group III and IV muscle afferents are probably important for determining motoneuron excitability during fatigue of elbow extensors, but that even for these muscles, other factors may also influence the motoneuron pool. Changes in other reflex pathways may be relevant. In particular, muscle spindle firing rates decline during fatiguing contractions and thus reduce facilitation to the motoneurons (Bongiovanni and Hagbarth, 1990; Macefield et al., 1991). Intrinsic motoneuronal properties also change with fatigue. In animals, when motoneurons are repetitively stimulated by sustained intracellular currents, many cells either reduce their discharge or stop firing (Kernell and Monster, 1982a,b; Spielmann et al., 1993; Sawczuk et al., 1997). Similarly, in humans, the synaptic drive necessary to maintain repetitive firing of single motor units increases during weak contractions (Johnson et al., 2004). Therefore, several mechanisms probably contribute to changes in motoneuron excitability observed during fatiguing contractions, and different mechanisms are likely to be more prominent at different times during a sustained contraction (Gandevia, 2001).

The current study provides evidence that motoneurons of extensor and flexor muscles of the human elbow are not uniformly affected by inputs from group III and IV afferents. Afferent inputs from homonymous and antagonist muscles inhibit extensor motoneurons, whereas motoneurons innervating flexors are facilitated. A corollary of these results is that if inhibitory influences from these afferents are more pronounced on extensor motoneurons then, all other things being equal, these muscles will require greater cortical output to generate a given force during fatigue. This suggests that rapid movements produced by coordinated flexor and extensor activity may be especially disturbed by fatigue.

Note added in proof. Throughout the text, we have indicated that the ischemia after the sustained contractions produced by the cuff maintained the discharge of populations of both group III and IV muscle afferents. A new paper by Hayes et al. (2006) strongly suggests that the population continuing to discharge will be composed largely of group IV muscle afferents. 


\section{References}

Andersen OK, Sonnenborg FA, Arendt-Nielsen L (1999) Modular organization of human leg withdrawal reflexes elicited by electrical stimulation of the foot sole. Muscle Nerve 22:1520-1530.

Bigland-Ritchie B, Johansson R, Lippold OC, Smith S, Woods JJ (1983) Changes in motoneurone firing rates during sustained maximal voluntary contractions. J Physiol (Lond) 340:335-346.

Bigland-Ritchie BR, Dawson NJ, Johansson RS, Lippold OC (1986) Reflex origin for the slowing of motoneurone firing rates in fatigue of human voluntary contractions. J Physiol (Lond) 379:451-459.

Bongiovanni LG, Hagbarth KE (1990) Tonic vibration reflexes elicited during fatigue from maximal voluntary contractions in man. J Physiol (Lond) 423:1-14.

Butler JE, Taylor JL, Gandevia SC (2003) Responses of human motoneurons to corticospinal stimulation during maximal voluntary contractions and ischemia. J Neurosci 23:10224-10230.

de Noordhout AM, Rapisarda G, Bogacz D, Gerard P, De Pasqua V, Pennisi G, Delwaide PJ (1999) Corticomotoneuronal synaptic connections in normal man: an electrophysiological study. Brain 122:1327-1340.

Duchateau J, Hainaut K (1993) Behaviour of short and long latency reflexes in fatigued human muscles. J Physiol (Lond) 471:787-799.

Eccles JC, Lundberg A (1959) Synaptic actions in motoneurones by afferents which may evoke the flexion reflex. Arch Ital Biol 97:199-221.

Gandevia SC (2001) Spinal and supraspinal factors in human muscle fatigue. Physiol Rev 81:1725-1789.

Gandevia SC, Macefield G, Burke D, McKenzie DK (1990) Voluntary activation of human motor axons in the absence of muscle afferent feedback. The control of the deafferented hand. Brain 113:1563-1581.

Gandevia SC, Petersen N, Butler JE, Taylor JL (1999) Impaired response of human motoneurones to corticospinal stimulation after voluntary exercise. J Physiol (Lond) 521:749-759.

Garland SJ (1991) Role of small diameter afferents in reflex inhibition during human muscle fatigue. J Physiol (Lond) 435:547-558.

Garland SJ, Kaufman MP (1995) Role of muscle afferents in the inhibition of motoneurons during fatigue. Adv Exp Med Biol 384:271-278.

Garland SJ, McComas AJ (1990) Reflex inhibition of human soleus muscle during fatigue. J Physiol (Lond) 429:17-27.

Grimby L, Hannerz J, Hedman B (1981) The fatigue and voluntary discharge properties of single motor units in man. J Physiol (Lond) 316:545-554.

Hagbarth KE (1960) Spinal withdrawal reflexes in the human lower limbs. J Neurol Neurosurg Psychiatry 23:222-227.

Hayes SG, Kindig AE, Kaufman MP (2006) Cyclooxygenase blockade attenuates the responses of group III and IV muscle afferents to dynamic exercise in cats. Am J Physiol Heart Circ Physiol, in press.

Holmqvist B, Lundberg A (1961) Differential supraspinal control of synaptic actions evoked by volleys in the flexion reflex afferents in alpha motoneurones. Acta Physiol Scand:1-15.

Johnson KV, Edwards SC, Van Tongeren C, Bawa P (2004) Properties of human motor units after prolonged activity at a constant firing rate. Exp Brain Res 154:479-487.

Jovanovic K, Anastasijevic R, Vuco J (1990) Reflex effects on gamma fusimotor neurones of chemically induced discharges in small-diameter muscle afferents in decerebrate cats. Brain Res 521:89-94.

Kalezic I, Bugaychenko LA, Kostyukov AI, Pilyavskii AI, Ljubisavljevic M, Windhorst U, Johansson H (2004) Fatigue-related depression of the feline monosynaptic gastrocnemius-soleus reflex. J Physiol (Lond) 556:283-296.

Kaufman MP, Forster HV (1996) Reflexes controlling circulatory, ventilatory and airway responses to exercise. In: Handbook of physiology. Exercise: regulation and integration of multiple systems, pp 381-447. Bethesda, MD: American Physiology Society.

Kernell D, Monster AW (1982a) Motoneurone properties and motor fatigue. An intracellular study of gastrocnemius motoneurones of the cat. Exp Brain Res 46:197-204.

Kernell D, Monster AW (1982b) Time course and properties of late adaptation in spinal motoneurones of the cat. Exp Brain Res 46:191-196.

Kniffki KD, Schomburg ED, Steffens H (1979) Synaptic responses of lumbar alpha-motoneurones to chemical algesic stimulation of skeletal muscle in spinal cats. Brain Res 160:549-552.

Kniffki KD, Schomburg ED, Steffens H (1980) Action of muscular group III and IV afferents on spinal locomotor activity in cat. Brain Res 186:445-447.

Kniffki KD, Schomburg ED, Steffens H (1981a) Synaptic effects from chem- ically activated fine muscle afferents upon alpha-motoneurones in decerebrate and spinal cats. Brain Res 206:361-370.

Kniffki KD, Schomburg ED, Steffens H (1981b) Convergence in segmental reflex pathways from fine muscle afferents and cutaneous or group II muscle afferents to alpha-motoneurones. Brain Res 218:342-346.

Kuchinad RA, Ivanova TD, Garland SJ (2004) Modulation of motor unit discharge rate and $\mathrm{H}$-reflex amplitude during submaximal fatigue of the human soleus muscle. Exp Brain Res 158:345-355.

Kukulka CG, Moore MA, Russell AG (1986) Changes in human alphamotoneuron excitability during sustained maximum isometric contractions. Neurosci Lett 68:327-333.

Levinsson A, Garwicz M, Schouenborg J (1999) Sensorimotor transformation in cat nociceptive withdrawal reflex system. Eur J Neurosci 11:4327-4332.

Ljubisavljevic M, Jovanovic K, Anastasijevic R (1992) Changes in discharge rate of fusimotor neurones provoked by fatiguing contractions of cat triceps surae muscles. J Physiol (Lond) 445:499-513.

Loscher WN, Cresswell AG, Thorstensson A (1996) Excitatory drive to the alpha-motoneuron pool during a fatiguing submaximal contraction in man. J Physiol (Lond) 491:271-280.

Macefield G, Hagbarth KE, Gorman R, Gandevia SC, Burke D (1991) Decline in spindle support to alpha-motoneurones during sustained voluntary contractions. J Physiol (Lond) 440:497-512.

Nielsen J, Petersen N (1994) Is presynaptic inhibition distributed to corticospinal fibres in man? J Physiol (Lond) 477:47-58.

Palmer E, Ashby P (1992) Corticospinal projections to upper limb motoneurones in humans. J Physiol (Lond) 448:397-412.

Petersen NT, Taylor JL, Gandevia SC (2002) The effect of electrical stimulation of the corticospinal tract on motor units of the human biceps brachii. J Physiol (Lond) 544:277-284.

Pettorossi VE, Della Torre G, Bortolami R, Brunetti O (1999) The role of capsaicin-sensitive muscle afferents in fatigue-induced modulation of the monosynaptic reflex in the rat. J Physiol (Lond) 515:599-607.

Rossi A, Dechii B, Ginanneschi F (1999) Presynaptic excitability changes of group Ia fibres to muscle nociceptive stimulation in humans. Brain Res 818:12-22.

Rossi A, Mazzocchio R, Decchi B (2003) Effect of chemically activated fine muscle afferents on spinal recurrent inhibition in humans. Clin Neurophysiol 114:279-287.

Rudomin P, Schmidt RF (1999) Presynaptic inhibition in the vertebrate spinal cord revisited. Exp Brain Res 129:1-37.

Sacco P, Newberry R, McFadden L, Brown T, McComas AJ (1997) Depression of human electromyographic activity by fatigue of a synergistic muscle. Muscle Nerve 20:710-717.

Sawczuk A, Powers RK, Binder MD (1997) Contribution of outward currents to spike-frequency adaptation in hypoglossal motoneurons of the rat. J Neurophysiol 78:2246-2253.

Schomburg ED, Steffens H, Kniffki KD (1999) Contribution of group III and IV muscle afferents to multisensorial spinal motor control in cats. Neurosci Res 33:195-206.

Spielmann JM, Laouris Y, Nordstrom MA, Robinson GA, Reinking RM, Stuart DG (1993) Adaptation of cat motoneurons to sustained and intermittent extracellular activation. J Physiol (Lond) 464:75-120.

Taylor JL, Gandevia SC (2004) Noninvasive stimulation of the human corticospinal tract. J Appl Physiol 96:1496-1503.

Taylor JL, Butler JE, Gandevia SC (1999) Altered responses of human elbow flexors to peripheral-nerve and cortical stimulation during a sustained maximal voluntary contraction. Exp Brain Res 127:108-115.

Taylor JL, Petersen N, Butler JE, Gandevia SC (2000) Ischaemia after exercise does not reduce responses of human motoneurones to cortical or corticospinal tract stimulation. J Physiol (Lond) 525:793-801.

Ugawa Y, Rothwell JC, Day BL, Thompson PD, Marsden CD (1991) Percutaneous electrical stimulation of corticospinal pathways at the level of the pyramidal decussation in humans. Ann Neurol 29:418-427.

Walton DM, Kuchinad RA, Ivanova TD, Garland SJ (2002) Reflex inhibition during muscle fatigue in endurance-trained and sedentary individuals. Eur J Appl Physiol 87:462-468.

Windhorst U, Kirmayer D, Soibelman F, Misri A, Rose R (1997) Effects of neurochemically excited group III-IV muscle afferents on motoneuron afterhyperpolarization. Neuroscience 76:915-929.

Woods JJ, Furbush F, Bigland-Ritchie B (1987) Evidence for a fatigueinduced reflex inhibition of motoneuron firing rates. J Neurophysiol 58: 125-137. 